Questions vives

\section{Questions Vives}

Recherches en éducation

Vol. $8 n^{\circ} 15$ | 2011

Égalité filles-garçons : où en est-on?

\title{
Les représentations sexuées des professions lors du processus de choix d'orientation vers les études de médecine chez les lycéen-ne-s de terminales scientifiques
}

The Gender-based representations of occupations and the orientation choice process for female and male fourth-year scientific secondary school students wish to pursue medical studies

\section{Céline Avenel}

\section{CpenEdition}

Journals

\section{Édition électronique}

URL : https://journals.openedition.org/questionsvives/732

DOI : 10.4000/questionsvives.732

ISBN : 978-2-8218-1090-7

ISSN : $1775-433 X$

Éditeur

Université Aix-Marseille (AMU)

\section{Édition imprimée}

Date de publication : 15 septembre 2011

ISBN : 978-2-912643-39-1

ISSN : 1635-4079

Référence électronique

Céline Avenel, «Les représentations sexuées des professions lors du processus de choix d'orientation vers les études de médecine chez les lycéen-ne-s de terminales scientifiques », Questions Vives [En ligne], Vol. 8 n¹5 | 2011, mis en ligne le 10 octobre 2011, consulté le 21 septembre 2021. URL : http:// journals.openedition.org/questionsvives/732; DOI : https://doi.org/10.4000/questionsvives.732

Ce document a été généré automatiquement le 21 septembre 2021.

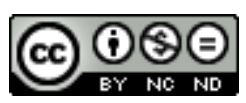

Questions Vives est mis à disposition selon les termes de la licence Creative Commons Attribution -

Pas d'Utilisation Commerciale - Pas de Modification 4.0 International. 


\section{Les représentations sexuées des} professions lors du processus de choix d'orientation vers les études de médecine chez les lycéen-ne-s de terminales scientifiques

The Gender-based representations of occupations and the orientation choice process for female and male fourth-year scientific secondary school students wish to pursue medical studies

Céline Avenel

\section{Introduction}

1 L'approche par l'orientation conduit à analyser les parcours individuels, familiaux et sociaux des lycéen-ne-s, donnant à voir les différences sexuées comme sociales. Cette ouverture d'accès aux filles, ce phénomène de féminisation massive des études de médecine n'entraîne pas mécaniquement d'évolutions dans les représentations sexuées des professions et spécialités médicales des lycéen-ne-s en terminales scientifiques. Il nous semble essentiel d'analyser les modalités et conséquences de cette féminisation des études de médecine sur les processus de choix d'orientation et professionnels des filles et des garçons. Les résultats de cette enquête démontrent une conséquence significative de cette féminisation: l'un des facteurs interagissant lors du développement de l'identité sexuée (les représentations sexuées des professions) reste prégnant et peut alors conditionner les choix d'orientation et les possibles de carrière des étudiant-e-s de médecine. 
2 La meilleure scolarité des filles ${ }^{1}$ ne se traduit pas dans leurs parcours scolaires. Elles restent surreprésentées en Lettres-Langues et Sciences et Humaines et Sociales (70 \% de filles) ainsi qu'en Médecine-Odontologie et Pharmacie (à plus de $65 \%$ ) à l'université ; mais aussi dans les écoles paramédicales ou préparant aux fonctions sociales ( $80 \%$ de filles), les écoles de vétérinaires ou encore les écoles de journalisme (avec toutes deux plus de $70 \%$ de filles) ${ }^{3}$. Certaines filières scientifiques et prestigieuses restent encore aujourd'hui largement masculines: $24 \%$ de filles en première année de Classe Préparatoire aux Grandes Ecoles (CPGE) maths, physique, sciences de l'ingénieur en 2006-07 (Pons, 2007), $26 \%$ en écoles d'ingénieur-e-s en 2008-09 (Filles et garçons sur le chemin de l'égalité de l'école à l'enseignement supérieur MESR, 2010) et moins de $15 \%$ pour l'informatique (Collet, 2006).

3 Pour les sociologues de l'orientation (Baudelot \& Establet, 1992; Duru-Bellat, 2004), l'intériorisation des rôles et stéréotypes de sexe, à l'école mais aussi à travers les attentes parentales, conditionne les représentations de soi et les choix d'orientation des filles et des garçons. Selon Duru-Bellat (2004), il existerait une rationalité et des stratégies de compromis dans les choix féminins d'orientation scolaire. Les filles font leur choix d'orientation en prenant compte, d'une manière réaliste, des facteurs objectifs du marché du travail et de la division du travail dans la famille et font preuve, en conséquence, d'une grande rationalité envers les stéréotypes dominants. Elles s'auto-contraignent et s'orientent ainsi massivement vers des filières et emplois, leur laissant du temps libre et/ ou pour la famille, mais généralement moins rémunérateurs. Même quand ils et elles envisagent des métiers scientifiques, garçons et filles se polarisent sur des domaines différents : sciences de l'ingénieur et informatique pour les premiers, santé, sciences de la vie ou chimie pour les secondes (Duru-Bellat, 1995).

4 Une recherche en Sciences de l'Education analyse le genre en tant que « ligne de partage nette au sein des usages de la scolarité secondaire et des orientations post-baccalauréat » (Jacques, 2003, p 65). Selon l'auteure, les représentations et intentions d'orientation postbaccalauréat féminines et masculines, à valeur scolaire identique, connaissent une différenciation horizontale (des caractéristiques différentes selon le sexe) et verticale (une hiérarchisation, les intentions féminines se concentrant dans les filières plus modestes). Les travaux de Lemaire (2005) apportent un éclairage sur la perception sexuée de la rentabilité des études. La grande diversité des modes d'exercice offrirait aux filles la possibilité de moduler leur activité. L'existence au contraire d'un modèle de référence de la réussite, avec celui des grandes écoles, amènerait les garçons à percevoir les études médicales comme moins rentables, en termes de réussite professionnelle (obtention d'un poste à responsabilités, réussite financière) comparativement à leur durée.

Des facteurs intervenant lors des choix d'orientation ont d'ores et déjà été démontrés par les psychologues (Vouillot, 1999, 2002 ; Mosconi \& Stevanovic, 2007). D’après Mosconi et Stevanovic (2007), les stéréotypes et rôles de sexe, définissant les qualités dites féminines et masculines et associant les compétences masculines à celles scientifiques, influencent largement les projets d'orientation et professionnels. Les filles, même quand elles envisagent ou choisissent un métier scientifique ou non-traditionnel, continuent à avoir des représentations stéréotypées du monde professionnel et conformes aux modèles traditionnels du rôle de genre. Vouillot (2002, p. 488) évoque que « les choix d'orientation sont instrumentalisés par la nécessité d'affirmation identitaire en tant que fille ou garçon ». D'après cette auteure, transgresser cette identité sexuée engendrerait un lourd coût identitaire et social pour les élèves. Il nous semble alors important d'analyser les 
représentations des professions chez les filles et garçons de terminales scientifiques, et notamment l'existence ou non d'une représentation sexuée des spécialités médicales, cela jouant un rôle lors des choix d'orientation vers les études supérieures.

6 L'accès des femmes aux études de médecine devient un phénomène massif à partir des années 90. Auparavant, elles ne représentent qu'un peu plus d'un tiers des étudiants en premier cycle des études médicales (Hardy-Dubernet, 2005) ; 26 \% des diplômé-e-s en 1975 et $40 \%$ en 1990. La proportion féminine va dépasser celle masculine en 1993-1994 avec $50,2 \%$ des effectifs tous cycles confondus (Lapeyre, 2006) et elles deviennent largement majoritaires (64\%) parmi les étudiants de première année en 2002 (Hérault \& Labarthe, 2003). Cette féminisation se poursuit jusqu'au troisième cycle de médecine avec 55,9\% d'étudiantes en Résidanat et 53,6 \% en Internat (pour l'année 2002) (Hérault \& Labarthe, 2003).

7 Il faut mettre tout d'abord ce phénomène en lien avec le contexte scolaire des années 60-70 : c'est-à-dire un investissement massif des filles dans le supérieur, impulsé par le développement de la mixité. Nous faisons l'hypothèse que ce mouvement général des filles qui investissent largement le supérieur avec de meilleures scolarités, ainsi que l'augmentation de bachelières scientifiques, n'a pas pour conséquence mécanique immédiate la féminisation des études de médecine. Cela n'explique pas tout de la féminisation depuis en trentaine d'années en France car si c'était le cas toutes les disciplines verraient leur part de filles, si ce n'est égaler celle des garçons, au moins s'en rapprocher. Or, ce n'est pas le cas, cette augmentation de bachelières scientifiques ne s'est pas diffusée égalitairement dans les filières scientifiques et techniques du supérieur (comme les CPGE maths-physique ou les écoles d'ingénieur-e-s). Les procédures d'orientation et les représentations sexuées des lycéen-ne-s conditionnent une présence féminine qui reste encore aujourd'hui inégale selon les filières et spécialités, précisément celles prestigieuses et scientifiques.

Cet article propose de s'interroger sur les évolutions des processus de choix d'orientation des étudiant-e-s dans l'enseignement supérieur, plus particulièrement vers les études de médecine dans un contexte de féminisation massive de ces études en France depuis une trentaine d'années, à travers l'analyse des représentations sexuées des lycéen-ne-s de terminales S. Les représentations des professions, opérant comme l'un des facteurs des choix post-baccalauréat des filles et des garçons; la féminisation d'une discipline historiquement masculine comme la médecine ${ }^{4}$ entrâ̂ne-t-elle une évolution de la division sexuée de ces représentations chez les lycéen-ne-s envisageant médecine?

9 Cet article se base sur une enquête par questionnaires réalisée dans le cadre de notre thèse en sciences de l'éducation sur les choix d'orientation vers les études de médecine chez les filles comparativement aux garçons. Cette enquête fut menée auprès de 176 lycéen-ne-s (92 filles et 84 garçons) de terminales scientifiques dans deux lycées urbains de l'académie de Montpellier durant l'année 2009. Cette méthode du questionnaire nous semblait la plus adaptée pour recueillir des données quantifiables sur les motifs d'orientation et projections des lycéen-e-s en terminale scientifique, mais aussi pour cette population peu disponible à cette période précédant les examens du baccalauréat. La terminale scientifique (S) nous semble un palier d'orientation essentiel à analyser : elle représente l'étape sélective conditionnant en grande partie l'accès aux études de médecine (92,2 \% des étudiant-e-s en premier cycle des études médicales première année (PCEM1) en 2002 possédait un bac S) (Hérault \& Labarthe, 2003). Il semble que c'est à ce moment du parcours scolaire qu'entrent en compte certains facteurs explicatifs des choix 
d'orientation des filles et des garçons vers les études de médecine. Cette enquête visait à analyser le processus d'orientation pratiquée en terminale de lycée sous l'angle du genre. C'est-à-dire observer les différences sexuées, mais aussi et surtout les facteurs de ces différences, lors des choix et projets d'orientation pour les études de médecine chez les filles comparativement aux garçons, dans un contexte de féminisation massive de ces études en France depuis une trentaine d'années.

\section{Les origines sociales des étudiant-e-s de l'échantillon} sociales et scolaires relativement proches. Les Catégories Socio-Professionnelles (CSP) favorisées sont les plus représentées chez les parents des lycéen-ne-s. Près de la moitié des pères sont présents dans les CSP «Cadres et professions intellectuelles supérieures » $(45,5 \%)$ et un tiers des mères appartient à la CSP «Cadres et professions intellectuelles supérieures » (33\%).

11 Concernant les filles et garçons, choisissant médecine dès la terminale $\mathrm{S}$, nous faisons la même observation de caractéristiques sociales similaires. Près de la moitié des pères, des lycéen-ne-s de l'échantillon choisissant médecine, sont présents dans les CSP «Cadres et professions intellectuelles supérieures» $(46,9 \%)$. L'origine sociale des «futur-e-s étudiant-e-s » de médecine est beaucoup plus élevée par rapport à la moyenne nationale : en 2009-10, toutes formations confondues, $30,5 \%$ des pères appartenaient à la CSP «Cadres et professions intellectuelles supérieures ». Les pères sont aussi représentés dans la CSP» Ouvriers » (12,5\%). L'ensemble des pères reste peu représenté dans les autres CSP, avec moins de $10 \%$. Les mères, des étudiants de l'échantillon choisissant médecine, se répartissent davantage dans les CSP, avec toujours une majorité exerçant en tant que «Cadres et professions intellectuelles supérieures » $(34,4 \%)$. Un quart est représentée dans les « Professions Intermédiaires » puis moins de $20 \%$ sont « employées ». A l'inverse des pères, $15,6 \%$ des mères n'ont pas d'activité professionnelle.

Une étude réalisée en 2002 (Hérault \& Labarthe) montre que la sélection a peu évolué entre 1992 et 2002, les cadres et professions intellectuelles supérieures restant les CSP les plus représentées chez les parents ( $44 \%$ des pères des étudiants en première année en 2002 étaient cadres ou occupaient une profession intellectuelle supérieure). Les résultats de notre enquête confirment l'actualité de ce constat. La féminisation n'est pas synonyme de démocratisation pour les études de médecine, les CSP favorisées restent les plus représentées chez les parents des futur-e-s étudiant-e-s de médecine.

\section{Les parcours scolaires des filles et garçons de terminales scientifiques}

Durant leur parcours scolaire, les filles de l'échantillon sont moins nombreuses que les garçons à redoubler pendant leur scolarité (13\% de filles contre $21,4 \%$ de garçons). Dans le questionnaire, nous demandions aux lycéen-ne-s leurs notes à l'examen écrit ainsi qu'à l'examen oral du baccalauréat de français. Le tableau 1 expose les notes obtenues par les filles et les garçons à l'examen écrit. 
Tableau $1:$ notes des élèves à l'épreuve écrite du baccalauréat de Français

\begin{tabular}{|l|l|l|l|}
\hline $\begin{array}{l}\text { Notes au Bac } \\
\text { de français (écrit) }\end{array}$ & S. masculin & S. féminin & TOTAL \\
\hline Moins de $5 / 20$ & $1,2 \%(1)$ & $0,0 \%(0)$ & $\mathbf{0 , 6} \%(\mathbf{1})$ \\
\hline De 5/20 à 9/20 & $40,5 \%(34)$ & $25,0 \%(23)$ & $\mathbf{3 2 , 4} \%(\mathbf{5 7})$ \\
\hline De $10 / 20$ à $15 / 20$ & $45,2 \%(38)$ & $63,0 \%(58)$ & $\mathbf{5 4 , 5} \%(\mathbf{9 6})$ \\
\hline Plus de $15 / 20$ & $13,1 \%(11)$ & $10,9 \%(10)$ & $\mathbf{1 1 , 9 \% ( 2 1 )}$ \\
\hline TOTAL & $\mathbf{1 0 0 , 0} \%(\mathbf{8 4})$ & $\mathbf{1 0 0 , 0} \%(\mathbf{9 1})$ & $\mathbf{1 0 0 , 0 \% ( 1 7 5 )}$ \\
\hline
\end{tabular}

La dépendance est peu significative. Chi2 $=7,07, \mathrm{ddl}=3,1-\mathrm{p}=93,03 \%$. Les valeurs du tableau sont les pourcentages en colonne établis sur 176 observations.

14 Il est vrai que l'analyse démontre un Chi2 non significatif, nous pouvons tout de même observer une tendance chez les filles à obtenir de meilleures notes à l'épreuve du Baccalauréat de français de première (à l'écrit comme à l'oral). Par exemple, à l'examen écrit : les garçons $(40,5 \%)$ ont davantage obtenu une note entre 5 et $9 / 20$ par rapport aux filles ( $25 \%)$. Et à l'inverse, les filles (63\%) ont plus souvent obtenu une note entre 10 et $15 / 20$ que les garçons ( $45 \%$ ). Et si l'on observe les très bonnes notes, au-dessus de $15 / 20$, il y a peu de différences entre les filles et les garçons $(13,1 \%$ de garçons et $10,9 \%$ de filles).

15 L'écart se creuse à l'inverse pour l'épreuve orale pour les notes supérieures à 15/20: $27,2 \%$ de filles ont obtenu une note supérieure à $15 / 20$ contre seulement $19 \%$ de garçons.

\section{Les choix d'orientation chez les filles et les garçons en terminales scientifiques}

Sur l'ensemble de l'échantillon, $55 \%$ des élèves évoquent le domaine de la santé comme choix après le bac. Les filles y sont effectivement plus nombreuses que les garçons : 59,8 \% de filles et $40,2 \%$ de garçons. De plus, parmi ces mêmes lycéen-ne-s évoquant la santé, $46,4 \%$ envisagent les études de médecine : avec une différence de 12 points entre les filles et les garçons puisque les filles représentent $56 \%$ de ces élèves contre $44 \%$ de garçons. Dans le questionnaire, une question concernait les lycéennes et lycéens ayant choisi l'Université après le baccalauréat, le tableau 2 présente les choix de filières cités par les filles et les garçons en terminale $\mathrm{S}$.

Tableau 2 : Les choix d'orientation à l'Université des lycéen-ne-s de terminales S

\begin{tabular}{|l|l|l|l|}
\hline $\begin{array}{l}\text { Université : choix } \\
\text { des études futures }\end{array}$ & S. masculin & S. féminin & TOTAL \\
\hline Sciences (non médicales) & $61,1 \%(22)$ & $38,9 \%(14)$ & $\mathbf{1 0 0 , 0 \% ( 3 6 )}$ \\
\hline \hline Médecine & $31,3 \%(10)$ & $68,8 \%(22)$ & $\mathbf{1 0 0 , 0} \%(\mathbf{3 2})$ \\
\hline Pharmacie & $10,0 \%(1)$ & $90,0 \%(9)$ & $\mathbf{1 0 0 , 0} \%(\mathbf{1 0})$ \\
\hline
\end{tabular}




\begin{tabular}{|l|l|l|l|}
\hline Droit & $27,3 \%(3)$ & $72,7 \%(8)$ & $\mathbf{1 0 0 , 0 \% ( 1 1 )}$ \\
\hline Informatique & $100,0 \%(7)$ & $0,0 \%(0)$ & $\mathbf{1 0 0 , 0 \% ( 7 )}$ \\
\hline Staps & $75,0 \%(3)$ & $25,0 \%(1)$ & $\mathbf{1 0 0 , 0 \% ( 4 )}$ \\
\hline \hline Autres & $66,7 \%(6)$ & $33,3 \%(3)$ & $\mathbf{1 0 0 , 0 \% ( 9 )}$ \\
\hline Non indiqué & $59,0 \%(23)$ & $41,0 \%(16)$ & $\mathbf{1 0 0 , 0} \%(\mathbf{3 9})$ \\
\hline Non concerné-e & $36,4 \%(12)$ & $63,6 \%(21)$ & $\mathbf{1 0 0 , 0} \%(\mathbf{3 3})$ \\
\hline \hline TOTAL & $\mathbf{4 7 , 7} \%(\mathbf{8 7})$ & $\mathbf{5 2 , 3} \%(\mathbf{9 4})$ & $\mathbf{1 0 0 , 0} \%(\mathbf{1 8 1})$ \\
\hline
\end{tabular}

La dépendance est très significative. chi2 = 27,43, $d d l=8,1-p=99,94 \%$. Les citations sont supérieures au nombre de lycéen-ne-s interrogé-e-s car les réponses pouvaient être multiples.

Parmi les élèves de l'échantillon définissant l'Université comme choix après le baccalauréat, $29,4 \%$ ont choisi la filière médecine. Parmi ces élèves, $68,8 \%$ sont des filles. Les souhaits d'orientation des filles vers médecine sont représentatifs de la situation nationale; à la rentrée 2008-09, 66,1\% des étudiant-e-s de PCEM1 étaient des filles (Repères et références statistiques, 2009).

Nos effectifs sont plus faibles en ce qui concerne la filière informatique et celle de pharmacie, et ne peuvent de ce fait rendre compte de l'ensemble des lycéen-ne-s de terminales $\mathrm{S}$, mais il est intéressant de les observer. Les filles et garçons s'y répartissent très nettement, $90 \%$ des élèves citant pharmacie sont des filles et à l'inverse l'informatique est la filière uniquement citée par des garçons dans cette enquête.

\section{La représentation des filières « masculines " persiste pour l'ensemble des lycéen-ne-s de terminale scientifique}

Concernant les vœux en CPGE scientifiques, en écoles d'ingénieur-e-s et dans la filière informatique, dont nos effectifs sont plus faibles, mais il nous semble intéressant de noter une tendance à la répartition sexuée dans les projections des lycéen-ne-s de l'échantillon. Si l'on observe précisément, ce sont davantage les garçons qui choisissent une orientation en CPGE scientifiques $(26,2 \%)$ et faiblement les filles $(9,8 \%$, les garçons citent de plus davantage les écoles d'ingénieur-e-s (10,7 \%) que les filles (seulement 3,3\%) et de même pour l'informatique qui est la filière uniquement citée par des garçons dans cette enquête. Cette répartition des projections post-baccalauréat féminines et masculines donne à voir une persistance des représentations des filières "masculines » à travers cette projection plus forte et fréquente des garçons en CPGE scientifiques, en écoles d'ingénieur-e-s ou encore dans la filière Informatique. Ces filières sont encore largement masculines aujourd'hui : $24 \%$ de filles en première année de CPGE maths, physique, sciences de l'ingénieur en 2006-07 (Pons, 2007), 26 \% en écoles d'ingénieur-e-s en 2008-09 (Filles et garçons sur le chemin de l'égalité de l'école à l'enseignement supérieur MESR, 2010) et moins de $15 \%$ pour l'informatique (Collet, 2006). 


\section{Les projets des filles, d'études comme professionnels, semblent plus « aboutis " que ceux des garçons}

Les filles autant que les garçons (autour de $48 \%$ pour chaque) estiment avoir des projets professionnels. Par contre, $37 \%$ des filles affirment avoir déjà choisi une profession (contre $25 \%$ des garçons), même en dehors des professions médicales. Les filles sont encore aujourd'hui toujours plus précises que les garçons concernant la façon dont elles envisagent l'avenir. Déjà en 2005, une étude sur la façon dont les lycéen-ne-s envisageaient l'avenir (Lemaire, 2005, p. 144) démontrait que «le projet professionnel tient une place importante pour les filles : près d'une bachelière sur deux (48\%) dit en terminale avoir une idée précise du métier qu'elle veut faire plus tard, alors que ce n'est le cas que de $38 \%$ des lycéens ».

Dans le questionnaire, nous demandions aux lycéen-ne-s s'ils et elles estimaient avoir des projets professionnels ou s'ils et elles avaient déjà défini une profession future. Les tableaux suivants 3 et 4 présentent respectivement les domaines professionnels cités par les filles et garçons ayant des projets de profession ainsi que les choix professionnels réalisés en terminale.

La division sexuée des professions se retrouve dans les projections professionnelles de l'ensemble des élèves de l'échantillon, tant pour celles et ceux ayant un projet que pour celles et ceux ayant déjà choisi une profession. Les professions les plus représentées pour les filles relèvent de la santé. Un quart des filles se projettent vers des professions dans les domaines médicaux, paramédicaux et biochimie; et près de $20 \%$ des garçons se projettent vers la profession d'ingénieur et dans le domaine de l'informatique. Il est vrai que l'analyse démontre un Chi2 non significatif, nous notons tout de même une tendance chez les filles et les garçons ayant défini une profession future dès la terminale. Quasiment une fille sur quatre $(22,8 \%)$ a déjà choisi une profession dans le domaine de la santé. Quant aux garçons, très peu (moins de $10 \%$ pour chaque modalité) ont cité une profession précise. 
Tableau. 3 : les projets de profession future des filles et des garçons de l'échantillon

\begin{tabular}{|l|l|l|l|}
\hline $\begin{array}{l}\text { Projets de } \\
\text { profession future }\end{array}$ & S. masculin & S. féminin & TOTAL \\
\hline \hline Ingénieur-e-s & $17,9 \%(15)$ & $8,7 \%(8)$ & $\mathbf{1 3 , 1} \%(\mathbf{2 3})$ \\
\hline $\begin{array}{l}\text { Médical-paramédical / } \\
\text { bio-chimie }\end{array}$ & $15,5 \%(13)$ & $25,0 \%(23)$ & $\mathbf{2 0 , 5} \%(\mathbf{3 6})$ \\
\hline Droit/lettres & $4,8 \%(4)$ & $4,3 \%(4)$ & $\mathbf{4 , 5} \%(\mathbf{8})$ \\
\hline Informatique & $6,0 \%(5)$ & $0,0 \%(0)$ & $\mathbf{2 , 8} \%(5)$ \\
\hline Enseignement primaire et secondaire & $4,8 \%(4)$ & $2,2 \%(2)$ & $\mathbf{3 , 4} \%(\mathbf{6})$ \\
\hline International & $1,2 \%(1)$ & $2,2 \%(2)$ & $\mathbf{1 , 7} \%(\mathbf{3})$ \\
\hline Autres & $7,1 \%(6)$ & $13,0 \%(12)$ & $\mathbf{1 0 , 2} \%(\mathbf{1 8})$ \\
\hline TOTAL & $\mathbf{1 0 0 , 0} \%(\mathbf{4 8})$ & $\mathbf{1 0 0 , 0} \%(\mathbf{5 1})$ & $\mathbf{1 0 0 , 0} \%(\mathbf{9 9})$ \\
\hline
\end{tabular}

La dépendance est significative. chi2 $=12,83, \mathrm{ddl}=5,1-\mathrm{p}=95,42 \%$. Les valeurs du tableau sont les pourcentages en colonne établis sur 176 observations

Tableau.4 : les choix de profession future des filles et des garçons de l'échantillon

\begin{tabular}{|l|l|l|l|}
\hline $\begin{array}{l}\text { Choix réalisés } \\
\text { de profession } \\
\text { future }\end{array}$ & S. masculin & S. féminin & TOTAL \\
\hline Ingénieur-e & $4,8 \%(4)$ & $2,2 \%(2)$ & $\mathbf{3 , 4} \%(6)$ \\
\hline Santé & $7,1 \%(6)$ & $22,8 \%(21)$ & $\mathbf{1 5 , 3} \%(27)$ \\
\hline Enseignement & $3,6 \%(3)$ & $5,4 \%(5)$ & $\mathbf{4 , 5} \%(\mathbf{8})$ \\
\hline Armée - police & $3,6 \%(3)$ & $3,3 \%(3)$ & $\mathbf{3 , 4} \%(\mathbf{6})$ \\
\hline \hline Autres & $8,3 \%(7)$ & $5,4 \%(5)$ & $\mathbf{6 , 8} \%(\mathbf{1 2})$ \\
\hline TOTAL & $\mathbf{1 0 0 , 0 \% ( 2 3 )}$ & $\mathbf{1 0 0 , 0} \%(\mathbf{3 6})$ & $\mathbf{1 0 0 , 0} \%(59)$ \\
\hline
\end{tabular}

La dépendance est peu significative. chi2 $=7,32, \mathrm{ddl}=4,1-p=88,03 \%$. Les valeurs du tableau sont les pourcentages en colonne établis sur 176 observations.

23 Nous nous sommes en outre intéressées aux lycéen-ne-s ayant déjà choisi les études de médecine en terminale $\mathrm{S}$. Cela réduit les effectifs observés mais nous tenons néanmoins à observer l'existence ou non de disparités dans les projets d'études et professionnels féminins et masculins. Les explications du choix pour médecine divergent entre ces filles et ces garçons : la moitié des filles considère choisir médecine pour pouvoir exercer une profession médicale qu'elles ont déjà définie (contre $30 \%$ des garçons), $40 \%$ des garçons citent ce choix par intérêt pour les professions médicales et paramédicales (contre $23 \%$ des filles) et nous observons que $20 \%$ des garçons évoquent le prestige de la profession (contre seulement $4,5 \%$ des filles). Nous remarquons un écart entre ces filles et ces garçons quant à leur choix de filière pour leur seconde année de médecine, suite au concours à la fin de la première année : elles sont plus nombreuses ( $91 \%$ de filles et $70 \%$ 
de garçons) à savoir, dès la terminale, ce qu'elles choisiront en PCEM2 (entre médecine, dentaire et sage-femme). Quasiment autant de filles comme de garçons estiment d'ailleurs y choisir médecine ( $70 \%$ de garçons et près de $65 \%$ de filles). Nous notons que les choix féminins sont plus diversifiés que ceux masculins, moins d'un quart des filles envisagent de choisir sage-femme et moins de $5 \%$ dentaire alors que les garçons mentionnent uniquement médecine.

Il existe quelques disparités concernant les projections professionnelles féminines et masculines. Tout d'abord, la moitié des garçons estime avoir des projets professionnels (pour $36 \%$ des filles) mais à l'inverse $60 \%$ des filles citent déjà définir leur profession future (ce n'est le cas que pour $40 \%$ des garçons). Si l'on observe les projets professionnels, autour de $40 \%$ des filles comme des garçons citent comme projet professionnel le domaine médical-paramédical et la biochimie, $10 \%$ des garçons mentionnent la profession d'ingénieur (pour à peine $5 \%$ des filles). Concernant les choix affirmés de profession future, il est intéressant d'observer que tous et toutes citent le domaine de la santé, avec une majorité de filles (60\%).

\section{Une persistance de la représentation sexuée des professions et spécialités médicales}

La répartition sexuée actuelle des professions se retrouve dans les représentations des lycéen-ne-s ayant choisi les études de médecine dès la terminale. Par exemple, nous avons demandé aux lycéen-ne-s d'estimer si les professions citées étaient exercées soit «en majorité par des femmes» / soit «en majorité par des hommes» / soit « indifféremment» c'est à dire exercées autant par une femme que par un homme (tableau 5).

Tableau. 5 : Estimation sexuée des professions chez les lycéen-ne-s ayant choisi médecine

\begin{tabular}{|l|l|l|l|l|}
\hline Profession & Femmes & Hommes & Indifféremment & TOTAL \\
\hline Enseignant-e (élémentaire) & $56,7 \%(17)$ & $0,0 \%(0)$ & $43,3 \%(13)$ & $\mathbf{1 0 0 , 0} \%(30)$ \\
\hline Professeur-e (collège/lycée) & $9,4 \%(3)$ & $3,1 \%(1)$ & $87,5 \%(28)$ & $\mathbf{1 0 0 , 0} \%(32)$ \\
\hline Infirmier-e & $84,4 \%(27)$ & $3,1 \%(1)$ & $12,5 \%(4)$ & $\mathbf{1 0 0 , 0} \%(32)$ \\
\hline Médecin & $3,1 \%(1)$ & $43,8 \%(14)$ & $53,1 \%(17)$ & $\mathbf{1 0 0 , 0} \%(32)$ \\
\hline Kinésithérapeute & $6,3 \%(2)$ & $43,8 \%(14)$ & $50,0 \%(16)$ & $\mathbf{1 0 0 , 0} \%(32)$ \\
\hline Vétérinaire & $9,7 \%(3)$ & $35,5 \%(11)$ & $54,8 \%(17)$ & $\mathbf{1 0 0 , 0} \%(31)$ \\
\hline Avocat-e & $9,7 \%(3)$ & $16,1 \%(5)$ & $74,2 \%(23)$ & $\mathbf{1 0 0 , 0} \%(31)$ \\
\hline Ingénieur-e & $0,0 \%(0)$ & $46,9 \%(15)$ & $53,1 \%(17)$ & $\mathbf{1 0 0 , 0} \%(32)$ \\
\hline Architecte & $3,1 \%(1)$ & $53,1 \%(17)$ & $43,8 \%(14)$ & $\mathbf{1 0 0 , 0} \%(32)$ \\
\hline \hline Ensemble & $\mathbf{2 0 , 1} \%(57)$ & $\mathbf{2 7 , 5} \%(\mathbf{7 8})$ & $\mathbf{5 2 , 5} \%(\mathbf{1 4 9})$ & $\mathbf{1 0 0 , 0 \% ( 2 8 4 )}$ \\
\hline
\end{tabular}

La dépendance est très significative. chi2 $=173,23, d d l=16,1-p=>99,99 \%$.

Ce tableau est construit sur la strate de population définie par le filtrage suivant : Université choix études futures = "médecine" 
rofessions d'Enseignant-e (dans le primaire) et Infirmier-e sont vues chez les lycéenne-s, ayant choisi les études de médecine, comme davantage exercés par des femmes (respectivement $56,7 \%$ et environ $84,4 \%$ ). La profession d'Architecte est estimée davantage exercée par des hommes (à $53 \%$ ). Les professions de Médecin, Kinésithérapeute, Vétérinaire et Ingénieur-e sont perçues comme étant exercées autant par des hommes que par des femmes (à plus de $50 \%$ ). Si l'on regarde ensuite la seconde estimation des lycéen-ne-s, en termes de pourcentage, ils et elles estiment que toutes ces professions sont exercées ensuite en majorité par des hommes (à plus de $35 \%$ pour vétérinaire et même près de $45 \%$ pour médecin, kinésithérapeute et ingénieur-e). C'est-àdire que les filles et les garçons estiment que ces professions sont soit exercées autant par des femmes que des hommes soit davantage par des hommes!

Nous avons posé la même question à ces lycéen-ne-s, d'estimer si les professions citées étaient exercées soit «en majorité par des femmes»/ soit «en majorité par des hommes" / soit "indifféremment», concernant les professions et les spécialités médicales (tableau 6).

Tableau. 6 : Estimation sexuée des professions de Santé chez les lycéen-ne-s ayant choisi médecine

\begin{tabular}{|l|l|l|l|l|}
\hline Profession & Femmes & Hommes & Indifféremment & TOTAL \\
\hline \hline Sage-femme & $100,0 \%(32)$ & $0,0 \%(0)$ & $0,0 \%(0)$ & $\mathbf{1 0 0 , 0} \%(32)$ \\
\hline Chirurgien-ne & $0,0 \%(0)$ & $84,4 \%(27)$ & $15,6 \%(5)$ & $\mathbf{1 0 0 , 0} \%(32)$ \\
\hline Pédiatre & $37,5 \%(12)$ & $12,5 \%(4)$ & $50,0 \%(16)$ & $\mathbf{1 0 0 , 0 \% ( 3 2 )}$ \\
\hline \hline Gynécologue & $34,4 \%(11)$ & $15,6 \%(5)$ & $50,0 \%(16)$ & $\mathbf{1 0 0 , 0 \% ( 3 2 )}$ \\
\hline Radiologue & $6,3 \%(2)$ & $34,4 \%(11)$ & $59,4 \%(19)$ & $\mathbf{1 0 0 , 0 \% ( 3 2 )}$ \\
\hline Neurologue & $0,0 \%(0)$ & $56,3 \%(18)$ & $43,8 \%(14)$ & $\mathbf{1 0 0 , 0 \% ( 3 2 )}$ \\
\hline \hline Ensemble & $\mathbf{2 9 , 7} \%(57)$ & $\mathbf{3 3 , 9} \%(65)$ & $\mathbf{3 6 , 5} \%(\mathbf{7 0})$ & $\mathbf{1 0 0 , 0 \% ( 1 9 2 )}$ \\
\hline
\end{tabular}

La dépendance est très significative. chi2 $=150,03^{\prime \prime} \mathrm{ddl}=10,1-\mathrm{p}=>99,99 \%$. Ce tableau est construit sur la strate de population définie par le filtrage suivant :Université choix études futures = "médecine"

Nous notons cette différenciation de façon identique: Sage-femme est vue comme exercée à $100 \%$ par les femmes. Chirurgien : par les hommes à près de $85 \%$. Et la même observation que précédemment pour Radiologue et Neurologue : près de la moitié des filles comme des garçons considèrent qu'elles sont exercées autant par les hommes que par les femmes. Viennent ensuite environ $40 \%$ des filles comme des garçons qui estiment qu'elles sont exercées davantage par des hommes (et même plus de $55 \%$ pour neurologue). Inversement, et toujours dans le sens de la division sexuée des professions, Pédiatre et Gynécologue : la moitié des lycéen-ne-s estiment qu'elles sont exercées autant par des femmes que par des hommes, viennent ensuite plus d'un tiers des lycéen-ne-s qui estiment que ces deux professions sont exercées davantage par des femmes.

Les filles sont plus nombreuses que les garçons à projeter de suivre des études de médecine après le bac. Elles semblent avoir des projets d'études et professionnel (même hors médecine) plus précis, aboutis que les garçons et pourtant leurs représentations sexuées persistent, des filières «typiquement » masculines (les filles n'estimant pas ou 
peu s'y engager) comme des professions médicales ou non. Ce qui montre bien que les représentations ne changent pas au même rythme que les évolutions (d'effectifs) dans le supérieur: les études de médecine se sont très largement féminisées cependant les représentations des autres filières du supérieur ainsi que des professions (médicales ou non) sont restées sexuées. Ce mouvement de féminisation n'a pas entraîné de modifications des représentations sexuées des professions chez les filles lors de leur choix post-baccalauréat, y compris chez les filles ayant déjà choisi les études de médecine en terminale.

\section{Conclusion}

Cette enquête par questionnaires a permis de caractériser la population des lycéen-ne-s de terminales $\mathrm{S}$ : les filles sont donc bien plus nombreuses que les garçons à projeter de suivre des études de médecine après le bac; l'existence de meilleures scolarités féminines ; une représentation moindre, affaiblie des capacités chez les filles ainsi qu'une persistance de la représentation des filières masculines.

31 Cette enquête s'appuie et confirme les résultats de recherches précédentes sur les choix d'orientation sexués vers les études supérieures, à travers cette prégnance de la représentation sexuée des professions et spécialités médicales et des projets féminins qui semblent plus aboutis, projets d'études comme professionnels. Mais les confirmer dans le cadre des études des études longues et scientifiques que représente médecine apporte un véritable paradoxe : les filles semblent avoir des projets d'études et professionnel plus précis que les garçons et pourtant leurs représentations sexuées persistent, des professions médicales ou non.

32 La féminisation des effectifs d'étudiant-e-s en médecine ne modifie pas la répartition sexuée déjà existante dans les filières longues et scientifiques; ni ne féminise les représentations des professions et spécialités médicales. Les filles sont plus nombreuses à s'engager vers des études de médecine, pour autant, les représentations ne changent pas au même rythme que les évolutions d'effectifs dans le supérieur. Malgré une mixité scolaire, impulsée en France dans les années 60-70, et un mouvement de féminisation des études de médecine depuis les années 90 , les stéréotypes de sexe restent prégnants lors de la socialisation, scolaire comme familiale, et lors du développement de l'identité sexuée des filles et des garçons.

Cette persistance des représentations sexuées chez les filles, y compris chez celles ayant choisi les études de médecine, s'analyse en lien avec les recherches développées sur la profession de médecin et des évolutions consécutives à cette féminisation en termes de pratique professionnelle (Lapeyre et Le Feuvre, 2005 ; Hardy-Dubernet, 2005). Lapeyre et Le Feuvre (2005) développent une analyse des effets de la dynamique du genre sur les pratiques des médecins, en termes du rapport à l'exercice professionnel et aux conditions d'offre des soins médicaux. Le phénomène de féminisation transforme la pratique médicale et engendre une évolution de l'éthos professionnel des médecins, qui n'est alors plus caractérisé par «le modèle de disponibilité permanente ». C'est-à-dire que la diversité des modes d'exercice médicaux, et surtout la possibilité de les moduler selon la vie familiale, dans le domaine de la santé (à travers la pratique libérale, le développement du temps partiel avec le cabinet de groupe chez les médecins généralistes, le salariat et l'ouverture de spécialités médicales aux femmes), qui concorde et s'est développée avec 
l'arrivée de nouvelles professionnelles, permettent aux filles de se projeter dans une profession permettant de concilier vie familiale et professionnelle.

Selon Hardy-Dubernet (2005, p. 48), cette féminisation des études de médecine se réalise dans un contexte bien spécifique, "Elles (les filles) adoptent un comportement de choix d'orientation qui ressemble à celui de leurs frères, en optant pour des études longues et sélectives, mais elles se conforment aussi à une tendance générale des étudiantes de leur sexe en se dirigeant vers le secteur de la santé ». L'emprise du genre, à travers les responsabilités féminines domestiques et familiales ainsi que les représentations sexuées traditionnelles des capacités féminines pour le soin, agit lors du choix d'orientation vers médecine chez les filles.

A l'inverse, et en réponse à une éventuelle «démasculinisation » de médecine, cette transformation de la pratique médicale conduirait les hommes à moins, voire difficilement, s'y identifier et se diriger vers d'autres domaines estimés plus rentables en termes de longueur d'études comme en termes financiers mais aussi symboliques. En conséquence, les garçons seraient davantage amener à s'orienter vers les CPGE ou les écoles d'ingénieur-e-s. Hardy-Dubernet évoque un "bouleversement d'une identité professionnelle de genre dans les deux sens, un métier qui perd son identité masculine en même temps qu'il se féminise » $(2005$, p. 49).

Le contexte des études de médecine s'avère spécifique: les effectifs se féminisent largement cependant les résultats de cette enquête démontrent la prégnance actuelle des représentations sexuées des professions, chez les futur-e-s étudiantes et étudiants en médecine, pouvant générer et perpétuer la division sexuée dans les choix d'orientation vers les études supérieures mais aussi dans les professions et spécialités médicales. Cette persistance des représentations sexuées fait écho à l'expression de "révolution respectueuse " de C.Marry, la répartition sexuée des filières et professions est en mouvement mais sans transformer les conceptions traditionnelles définissant les domaines du masculin et du féminin.

\section{BIBLIOGRAPHIE}

Baudelot, C., \& Establet, R. (1992). Allez les filles! Paris : Seuil.

Collet, I. (2006). L'informatique a-t-elle un sexe, Hackers, mythes et réalités. Paris : L'Harmattan.

Dall'Ava-Santucci, J. (2004). Des sorcières aux mandarines. Histoire des femmes médecins. Paris :

Calmann-Lévy.

Duru-Bellat, M. (1995). Filles et garçons à l'école, approches sociologiques et pycho-sociales.

Revue française de pédagogie, 110, 75-109.

Duru-Bellat, M. (2004). L'école des filles. Quelle formation pour quels rôles sociaux ? Paris :

L'Harmattan.

Hardy-Dubernet, A.-C. (2005). Femmes en médecine : vers un nouveau partage des professions? Revue française des affaires sociales, 59(1), 35-58. 
Hérault \& Labarthe (2003). Les étudiants inscrits en médecine en janvier 2002. Note d'information MJER, 48.

Jacques, M.-H. (2003). Garçons et filles de classes de terminales : le filtre sexué des représentations du cursus et des intentions d'orientation post-baccalauréat. Carrefours de l'éducation, 15, 62-81

Lapeyre, N. (2006). Les professions face aux enjeux de la féminisation. Toulouse : Octarès.

Lapeyre, N., \& Le Feuvre, N. (2005). Féminisation du corps médical et dynamiques professionnelles dans le champ de la santé, Revue française des affaires sociales, 59(1), 59-81.

Lemaire, S. (2005). Les premiers bacheliers du panel : aspirations, image de soi et choix d'orientation. Education et formations, 72, 137-153.

MEN (2009). Repères et références statistiques. Paris : La documentation française.

MESR(2010). Filles et garçons sur le chemin de l'égalité de l'école à l'enseignement supérieur.

Mosconi, N., \& Stevanovic, B. (2007). Genre et avenir. Les représentations des métiers chez les adolescentes et les adolescents. Paris : L'Harmattan.

Pons (2007). Les étudiants en classe préparatoire aux grandes écoles. Note d'information MESR, 37.

Vouillot, F. (1999). Orientation : le reflet des rôles de sexe. In F. Vouillot (Ed.), Filles et garçons à l'école : une égalité à construire (pp. 81-90).Paris : MEN, MESRT, CNDP.

Vouillot, F. (2002). Construction et affirmation de l'identité sexuée et sexuelle : éléments d'une analyse de la division sexuée de l'orientation : présentation. L'orientation scolaire et professionnelle, 31(4), 485-494.

\section{NOTES}

1. En 2008 , toutes sections confondues, $89 \%$ des filles qui se sont présentées au baccalauréat général l'ont obtenu, contre $87 \%$ des garçons (Filles et garçons sur le chemin de l'égalité de l'école à l'enseignement supérieur MESR, 2010).

2. Repères et références statistiques, édition 2010.

3. Filles et garçons sur le chemin de l'égalité de l'école à l'enseignement supérieur MESR, 2010.

4. Les femmes, étant interdites dès l'ouverture de l'Université, furent exclues des études et de l'exercice de la médecine dès le Moyen-âge (Dall'Ava-Santucci, 2004).

\section{RÉSUMÉS}

Cet article propose d'interroger les évolutions des processus de choix d'orientation des étudiante-s dans l'enseignement supérieur à travers les études de médecine, dans un contexte de féminisation massive de ces études en France depuis une trentaine d'années. Les résultats d'une enquête par questionnaires, menée auprès de 176 lycéen-ne-s de terminales scientifiques de l'académie de Montpellier durant l'année 2009, démontrent la persistance des représentations 
sexuées des professions et spécialités médicales lors du choix d'orientation vers les études de médecine.

The aim of this article is to study the evolution of the curriculum orientation of students of both genders in higher studies in a context of mass feminization of medical studies in France for the last thirty years. This article is based on the results of a survey by questionnaire of 176 fourthyear scientific students ( 92 girls and 84 boys) in two urban secondary schools of the academy of Montpellier in 2009. The results of our study show the continuing influence of gender representations of professions and specialized medical field orientations in orientation choices.

\section{INDEX}

Mots-clés : choix et projet d'orientation, féminisation des études de médecine, genre, représentations sexuées

Keywords : feminization, gender, gender conceptions, medical studies, orientation choices

\section{AUTEUR}

\section{CÉLINE AVENEL}

Doctorante en Sciences de l'éducation - Université de Montpellier 3 - LIRDEF (EA 3749) 